\title{
Healthcare Game Design: Behavioral Modeling of Serious Gaming Design for Children with Chronic Diseases
}

\author{
Hadi Kharrazi, Anthony Faiola, and Joseph Defazio \\ Indiana University Purdue University Indianapolis, School of Informatics \\ 535 W. Michigan St., Indianapolis, Indiana 46202 \\ \{kharrazi, afaiola, jdefazio\}@iupui.edu
}

\begin{abstract}
This article introduces the design principles of serious games for chronic patients based on behavioral models. First, key features of the targeted chronic condition (Diabetes) are explained. Then, the role of psychological behavioral models in the management of chronic conditions is covered. After a short review of the existing health focused games, two recent health games that are developed based on behavioral models are overviewed in more detail. Furthermore, design principles and usability issues regarding the creation of these health games are discussed. Finally, the authors conclude that designing healthcare games based on behavioral models can increase the usability of the game in order to improve the effectiveness of the game's desired healthcare outcomes.
\end{abstract}

Keywords: Serious Gaming, Diabetes, Hypoglycemia, Behavioral Modeling, Patient Empowerment, Compliance to Treatment, Adolescent Diabetic Drivers.

\section{Introduction}

\subsection{Diabetes}

The paper argues for the use of serious gaming as a leverage to help adolescents cope with the consequences of Type 1 Diabetes (T1D) and Type 2 Diabetes (T2D). Hypoglycemia is a form of low blood glucose that occurs in diabetic patients and has the potential to be severe and cause fatal accidents. Proper patient behavior is crucial for long term management of diabetes and prevention of its complications. Behavioral models in healthcare, such as the Health Belief Model, are increasingly viewed as being useful to improve the behavior of patients with such chronic conditions as diabetes. While traditional patient empowerment methods can be helpful in achieving higher compliance, adolescents need a much higher level of motivation. The increase of adolescents acquiring T2D [1] has led to pediatricians increasingly looking to disseminating health risk information and healthcare messages directly to adolescents through cell phones, videogames, and Websites. This is because adolescents are heavy users of media technologies that are rich in content and easy to deliver, with information presented in formats that are both highly entertaining and highly interactive. For example, there is substantial anecdotal evidence from healthcare 
providers that they must compete with computers, games, and social networking sites (e.g., Facebook ${ }^{\Theta}$ and MySpace ${ }^{\Theta}$ ) for their young patients' attention. For this reason, the National Institute of Diabetes, Digestive and Kidney Diseases has funded the development and distribution of game like "Escape from Diab" and "Nanoswarm" as part of a national study to determine if school age children can be motivated to improve their eating and exercise habits [2][3]. Other researchers are experimenting with healthcare messages delivered through iPods, MP3 players, and other audiocentric devices.

Real challenges, however, include the fact that health professionals tend to see the "production of multimedia teaching tools as important, but have difficulty identifying funds for development. The market for such tools has yet to become clear. Insurance companies have occasionally reimbursed for patient education, but have not traditionally developed the educational tools themselves."[4].

At the same time, there is a "serious gaming" movement with a substantial community interested in health-related game design and development. While there is almost universal agreement that engaging formats have a better chance of gaining attention, conclusive evidence is limited as to whether they actually change behavior. For example, a study of two highly interactive health Websites suggested that challenging interactivity can significantly affect comprehension and attitudes toward health Websites [5]. Moreover, serious games that both challenge and educate have shown preliminary results that suggest that they are effective in increasing compliance in diabetes treatment [6]. However developers of serious games for healthcare must understand the implications of new media as a complex domain that includes gaming technologies, usability engineering, and gaming and learning theory. As Winn (2008) suggests [7], "Designing effective, engaging serious games requires theoretical understanding of learning, cognition, emotion, and play". The authors argue that serious games should be assessed as useful tools to increase compliance to diabetes treatment and benefits to the effects of preventing hypoglycemia while driving.

\subsection{Behavioral Models}

\section{The Health Belief Model (HBM)}

HBM was originally conceived in 1952 as a systematic method to identify, explain, and predict preventive health behavior; it is regarded as the genesis of systematic and theory-based research in health behavior [8][9]. The model helped researchers to focus on the relationship between health behaviors, related practices, as well as the utilization of health services. HBM outlines that an individual's intention to engage in a health behavior is determined by general health values, specific health beliefs about vulnerability to a particular health threat, and beliefs about the consequences of the health problem. HBM can be used to evaluate or influence an individual's behavioral changes in regard to a particular health condition (Fig. 1).

The importance of this model relies in the fact that the key factors that are thought to influence behavior are modifiable through intervention. It includes knowledge about the condition, but maintains that knowledge alone is insufficient to change behaviors. This is in contrast to other theories that explain behaviors solely by means of non-modifiable factors such as age, race/ethnicity, socioeconomic status or factors that are very difficult to change such as psychopathology. 


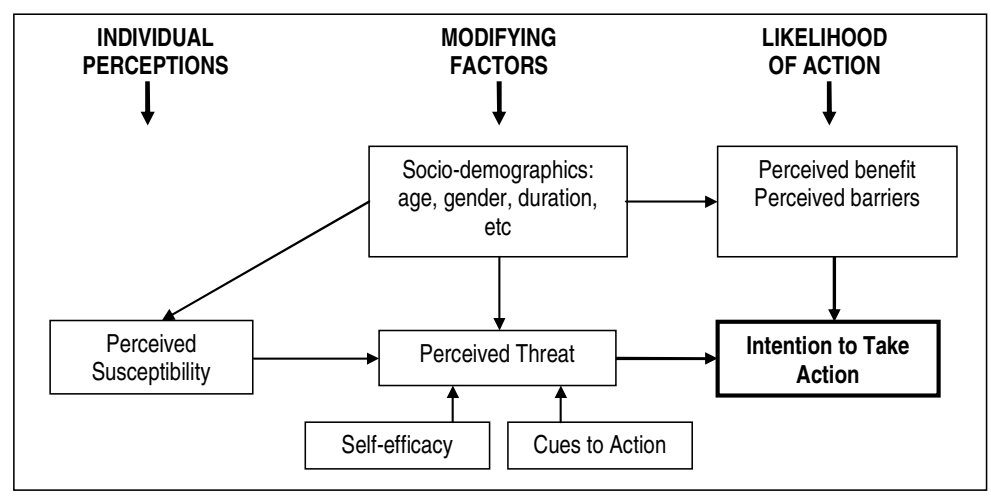

Fig. 1. The Health Belief Model

\section{The Planned Behavioral Model (PBM)}

According to the theory of planned behavior, human action is guided by three components: "beliefs about the likely outcomes of the behavior and the evaluations of these outcomes (behavioral beliefs), beliefs about the normative expectations of others and motivation to comply with these expectations (normative beliefs), and beliefs about the presence of factors that may facilitate or impede performance of the behavior and the perceived power of these factors (control beliefs)" [10]. Indeed behavioral beliefs make a behavior favorable or unfavorable (attitude), normative beliefs produce a social pressure (subjective norm) and control beliefs show the intensity of control factors (perceived behavioral control). Fig. 2 represents a schematic view of the theory.

Based on this model, behavioral changes can be achieved by targeting any of the factors: attitudes, subjective norms, or perceptions of behavioral control. The result of such an intervention should produce changes in behavioral intentions and, given adequate control over the behavior, the new intentions will be carried out under appropriate circumstances [11]. The common approach in behavioral models is determining the major elements that can affect participant's (patient's) behavior substantially enough to improve their behavior toward their chronic condition.

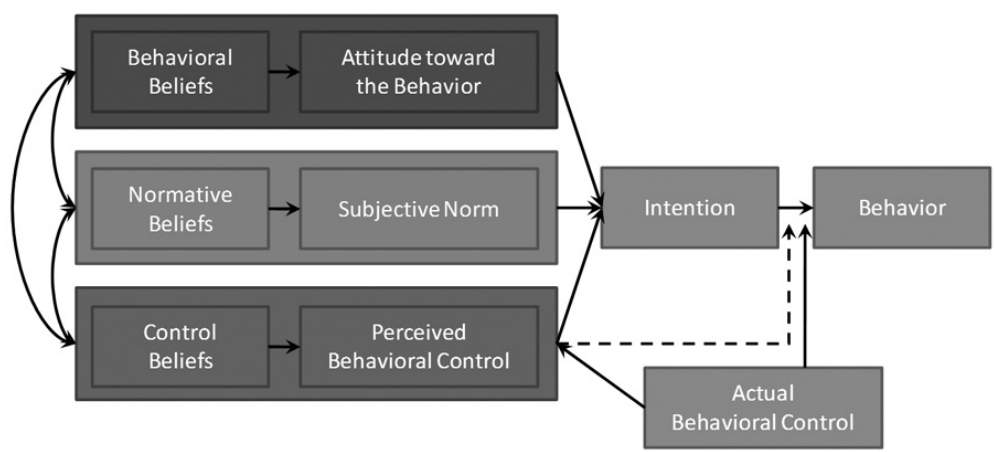

Fig. 2. The Planned Behavioral Model [10] 


\section{Background}

\subsection{Patient Empowerment and Serious Gaming}

Diabetic educators have defined patient empowerment as "the discovery and development of one's inherent capacity to be responsible for one's own life. [And the necessity to] influence their own behavior and that of others to improve the quality of their lives" [12]. The major hurdle in traditional methods of patient empowerment is motivating patients to change their behavior and maintain that change. This problem becomes even more pronounced in adolescents.

One of the most significant factors in better health outcomes for children with long term or chronic disorders is empowering the patients to consistently comply with the treatment regimen, even when they are not experiencing direct effects. Ubiquitous digital games, now considered a mass medium [13], can be exploited to achieve these health objectives. The factors that make digital games so engaging can be applied successfully in health contexts where motivation and engagement are necessary for the management of chronic conditions.

Today, the world of computer gaming has expanded rapidly to include applications other than entertainment, such as education. This new genre of games is named "serious games" [14][15][16]. As such, games differ in their use of visual, textual, and auditory channels for feedback, scaffolding challenges, visible goal indicators, overviews and schematics, and ease of learning [17]. This is in stark contrast to the typical "casual games" that are entertainment-centric. The process of learning how to play, how to improve skills, and how to succeed is much more natural in most games, and for applications of diabetes self-management and nutrition education, they continue to show promise.

\subsection{Examples of Serious Games for Type 1 Diabetes}

"Packy and Marlon" is a side-scrolling adventure game (played on a Super Nintendo console) that helps children and teens with type 1 diabetes self-management. "Glucoboy" is a glucometer that plugs into several video game devices, e.g., Nintendo Advance ${ }^{\circledR}$ and Nintendo Game Boy ${ }^{\circledR}$. The game is intended to encourage blood sugar monitoring and testing in kids with diabetes, with an advanced blood glucose meter that is extremely accurate and highly precise. "Escape from Diab" is a serious videogame adventure in healthy eating and exercise, with the goal to help prevent kids from becoming obese and developing diabetes and other related illnesses. "Nanoswarm" is another game that is about diabetes education that provides the player with a rich, immersive, interactive game. The game's developers hope that through role-playing and a blend of sci-fi action, they can bring the player to change their behavior toward a healthier life style. Developed by the USDA, the "MyPyramid Blast Off" is an interactive online game where kids can reach Planet Power by fueling their rocket with food and physical activity. Fuel tanks for each food group help children keep track of how their choices fit into MyPyramid. "Insulot" [18] is a cellular phone-based health education learning tool developed for T1D children in Japan. The game is intended to encourage, motivate, and boost the confidence of T1D patients, with outcomes to change behavior. "FatWorld" is a game about the politics of nutrition; a game the challenges the player to decide to be fit or fat, to live or to die. 


\section{Current Research}

\subsection{Serious Games and T1D Compliance to Treatment}

In a study with T1D patients, a game framework based on the Theory of Planned Behavior was used to increase the compliance/adherence rate to treatment. The main research question of this study is "Can games improve adherence to treatment in children with chronic diseases?" Parents reported the compliance rates of their children and the children (patients) were awarded in the game based on their compliance to treatment. Health points collected by compliance can be used in the game to buy new items or play additional mini-games. The study showed a significant increase in compliance rates [6]. Fig. 3 includes some screenshots of the game.
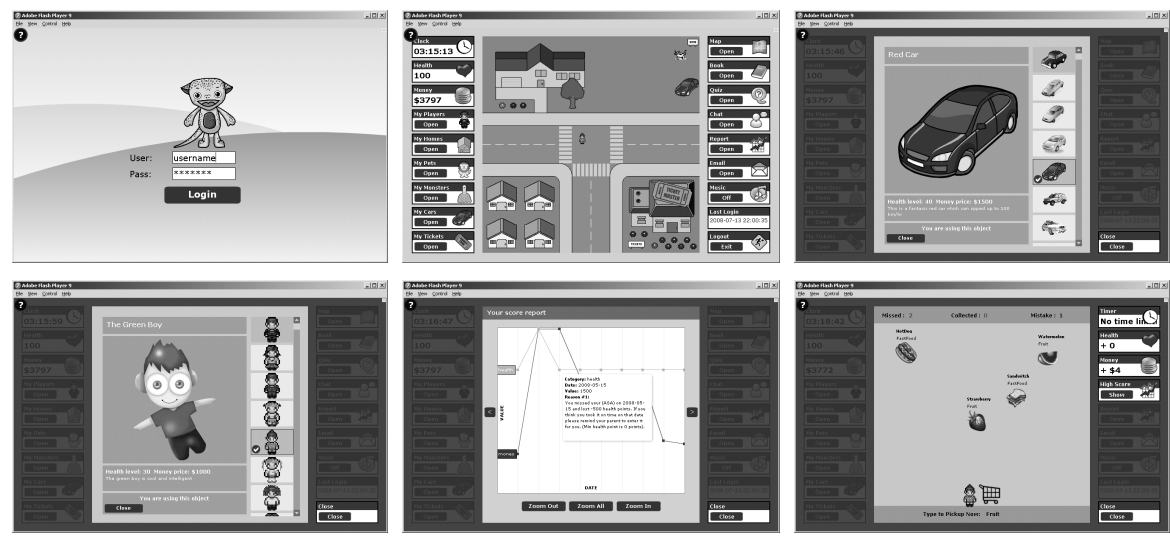

Fig. 3. Screenshots of a serious game designed for Type 1 Diabetic patients [6]. Left to right: Top - the login screen; main interface; using scores to buy new items. Bottom - using scores to change options; compliance report for children, and Playing mini-games based on points.

\section{Conceptual Framework}

Theoretical models of empowerment, compliance and behavioral changes were broken down into its elements and possible matching game elements were identified for each of them. Many of the conceptual elements in empowerment, compliance and behavioral models overlapped each other and therefore were removed. For example, control factors are included in multiple models such as PBM, compliance models and patient empowerment models.

Then, the elements that have close conceptual meaning were merged. For example, normative belief in PBM is close to family and peer support in contextual-behavioral model for patient empowerment. The remaining list was purged based on multiple focus group results. For example, none of the focus groups showed an interest in knowing the side effects of a given medication in the game. The final list of elements was refined based on the practicality of the game elements that are matched with the behavioral models.

After final considerations and defining the practical solutions some feasible elements were identified and chosen as the key functional game elements that map to 
different factors in empowerment, compliance and behavioral change models. The final framework included: Knowledge base and educational content, Motivational factors such as Pointing systems and Virtual mentoring systems, Peer Pressure and Measurement tools such as Questionnaires and Compliance reports.

\section{Usability Study}

Usability of this game framework is measured by questionnaires. Some of the questions are Likert-scale while some others are comment based. Usability questions are categorized as: Efficiency which reflects the goal of the game in affecting compliance; Satisfaction which indicates how much fun the game has been; Learnability that shows how easy learning the game was; and Memorability which refers to the ease of memorizing game features; and finally Error that measures the rate of error in the game's functionality (Fig. 4):

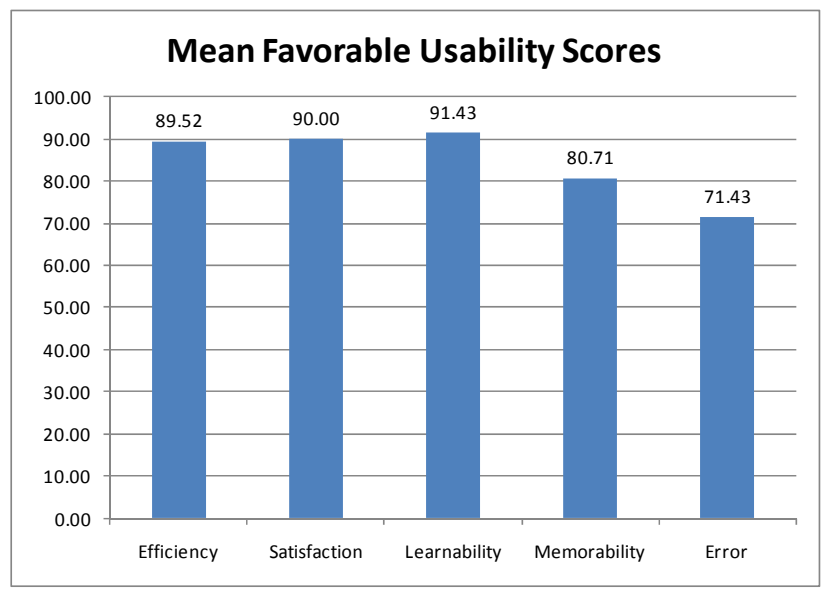

Fig. 4. Mean usability score based on different categories

\subsection{Game Pilot Study for Adolescent Diabetic Drivers}

The pilot study, titled, Testing for Hypoglycemia While Driving, is an educational game aimed at adolescent diabetic drivers with T1D. This study was a required first step (Phase 1) in developing a larger (Phase 2) and more intensive intervention within the context of a computer-assisted blood glucose awareness education program. As such, the resources required to develop and implement a large-scale intervention to change behaviors was beyond the scope of what is outlined here, i.e., in Phase 1. The purpose of the study, however, is to change adolescents' and parents' attitudes about hypoglycemia and driving, as well as to change their knowledge of perceived susceptibility, perceived seriousness and threat, perceived benefits to taking action, and barriers to taking action. Finally, the ultimate intent is to decrease the risk of hypoglycemia while driving among adolescents with diabetes. 


\section{Phase 1: Gaming Pilot Design and Testing Stage}

In Phase 1, the authors modified and adapted the Cox intervention to a brief educational intervention to be used on a laptop computer [19][20]. In this phase, gaming scenarios were designed and developed, along with the HBM attitudinal and behavioral measures. The theoretical underpinning of game design was HBM, because it addresses the understanding that: (1) a person will take a health-related action if they feel that a negative health condition, such as low blood sugar, can be avoided, (2) a person will have a positive expectation that adhering to the recommended action will avoid a negative health condition, and (3) a person believes that they can take a recommended health action with a successful outcome.

Four learning scenarios were developed during the analysis and design stages of the project (Fig. 5). For example, Scenario 1 allowed the participant to make choices prior to and during the drive to school. Scenario 2 allowed the participant to make choices prior to and during the drive home.

The theoretical grounding for the multimedia design and testing of Phase 1 was the Multimedia Development Model (MDM) [21], which was derived from the ADDIE (Analysis, Design, Development, Implementation, and Evaluation) instructional model. As reflected in ADDIE, MDM provided an iterative process that allowed the designers to re-visit each development stage during production. An important aspect of the model is its non-linear framework that allowed a repeatedly review and revise of the product. During the analysis phase, plans for content creation were reviewed. During the design phase, flowcharts and storyboards were developed using prewritten scenarios. Formative reviews of each asset and it's functionality within each scenario helped to ensure the quality and standard of the design during production.

HBM guided information presentation (susceptibility/severity of condition, benefits of behavioral change) and ultimate performance (behavioral cues) as the foundation for this design. During the analysis and design phases, graphical, textual, audio, and narrative assets were created. Once created, assets were incorporated into

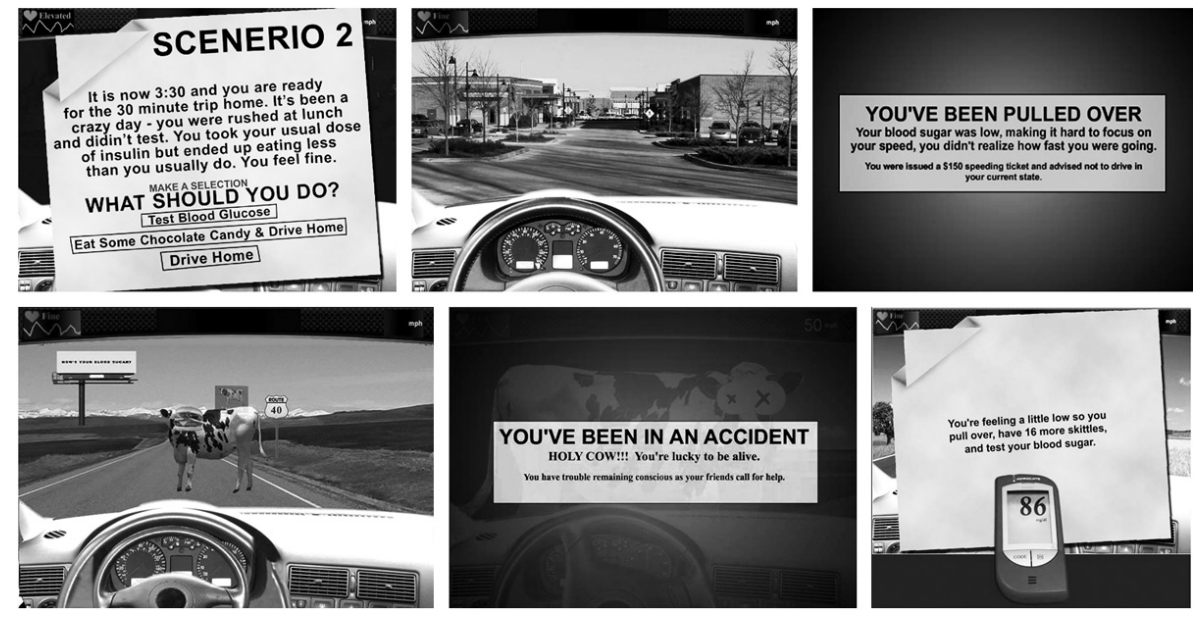

Fig. 5. Scenario Two - Phase 1 of Educational Gaming for Adolescent Drivers with Diabetes 
the design and reviewed for appearance, appropriateness, functionality, and fit. The final phase of the design cycle implemented usability testing on several subjects to ensure functionality and flawless execution of the design.

\section{Usability Testing}

Adolescent participants were recruited and the education game modules were tested for content clarity and usability through a usability and user experience evaluation of the product. After the usability findings were collected and analyzed the appropriate changes were made. The following figure (Fig. 6) depicts the average score for the participants' responses to the usability questions (Likert-scale questions):

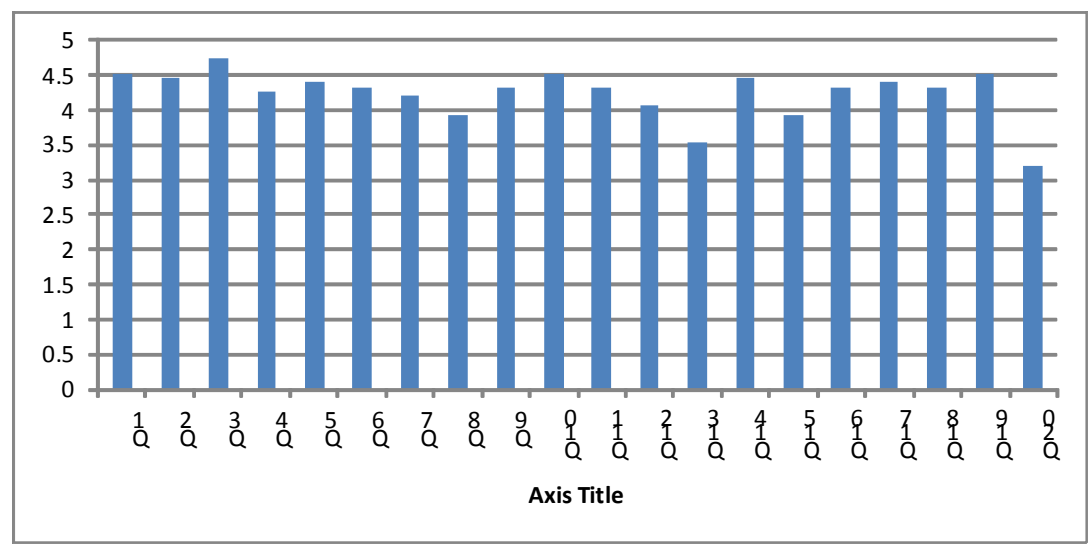

Fig. 6. Average score of responses to usability questions

\section{Phase 2: Gaming Pilot Testing Stage}

In Phase 2, researchers will administer the gaming modules to 150 adolescents ages $15-18$ and one of their parents. Information on behavior, attitude, and knowledge acquisition will be collected and analyzed to better predict the likelihood that adolescents and their parents will take action concerning the existing health condition.

\section{Conclusion}

Interactive media, including serious games, are becoming an inescapable part of our everyday life. With the emerging digital gaming culture, serious gaming will become an increasingly vital part of healthcare education for upcoming generations. Advanced interactive user interfaces now provide new opportunities for serious games that can be used not only to educate younger patients about their disease, but also to empower them for a positive change in their behavior both now and into full adulthood.

Chronic care constitutes a large slice of national healthcare expenses. Serious gaming can be an economical solution to educating, motivating, tracking, and empowering chronic patients for long term management of diabetes and prevention of complications. Serious games can lower not only the cost of chronic diseases but also 
the additional costs associated with side effects. Moreover, serious games aimed at health education do not involve large expenses as minimum healthcare staff is required to interact with the patient.

In an ideal game, the game's behavioral model can be integrated into both the patient's personal health record and the hospital/doctor's health record system. The health record integration can help the system to reward the patient in the game based on actual improved health results, and it can also help the physician/healthcare staff to track the patient's compliance to treatment; including other age groups such as adults and elderly patients. Artificial intelligence agents can be used to alert both the patient and the physician if the health status deteriorates. Moreover, different behavioral change models should be experimented with to find the most suitable model for an interactive patient empowerment approach.

Managing hypoglycemia in adolescents is critical due to their lack of insight into their disease and the lower compliance rate to treatment. Behavioral models have been used to change the behavior of the patients toward their disease and to improve their adherence to treatment. In this article, Planned Behavioral Model and Health Belief Model were the underlying behavioral models. Two studies were conducted: one to increase compliance to treatment in T1D and another to reduce severe driving consequences of hypoglycemia ion T1D. Both studies showed significant results in usability measures of effectiveness and satisfaction.

In summary, advancements in gaming technology, social and health informatics, and interaction design principles and practices, have all provided new knowledge and approaches to facilitating patient education and patient empowerment to achieve higher compliance [22][6].

\section{Acknowledgement}

Portions of this chapter are used with permission from Dr. Donald Orr, Adolescent Medicine, Indiana University, School of Medicine, Indianapolis, Indiana.

\section{References}

1. Hannon, T., Rao, G., Arslanian, S.: Childhood Obesity and Type 2 Diabetes Mellitus. Pediatrics 116(2), 473-480 (2005)

2. Baranowski, T., Archimage, I.: Escape from Diab (Accessed, 2008)

3. Lee, C.: New Video Games Not Just for Fun. Washington Post (October 2006)

4. Hayes, B., Aspray, W.: The Informatics of Diabetes: A Research Agenda for the Socially and Institutionally Sensitive Use of Information Technology to Improve Healthcare. MIT Press, Cambridge (2009) (in press)

5. Lustria, M.: Can interactivity make a difference? Effects of interactivity on the comprehension of and attitudes toward online health content. Journal of the American Society for Information Science and Technology 58(6), 766-776 (2007)

6. Kharrazi, H., Watters, C., Oore, S.: Improving behavioral stages in children by adaptive applications. Journal on Information Technology in Healthcare 6(1) (2008) 
7. Winn, B.: The Heart of Serious Game Design. In: Annual meeting of the International Communication Association, San Francisco, CA (2008),

http://www.allacademic.com/meta/p170925_index.html

8. Hochbaum, G.: Health Belief Model: Why People Seek Diagnostic X-rays. Public Health Reports 71, 377-380 (1956)

9. Hochbaum, G.M., Sorenson, J.R., Lorig, K.: Theory in Health Education Practice. Health Education Quarterly 19(3), 293-313 (1992)

10. Ajzen, I.: The Theory of Planned Behavior. Organizational Behavior and Human Decision Processes 2(50), 179-211 (1991)

11. Ajzen, I.: From Intentions to Actions: A Theory of Planned Behavior, 11-39 (1985)

12. Funnell, M.M., Anderson, R.M., Arnold, M.S.: Empowerment: a winning model for diabetes care. Practical Diabetol 10, 15-18 (1991)

13. Wolf, M.: The Medium of the Video Game. University of Austin Press, Austin, USA (2001)

14. Blackman, S.: Serious games and less! ACM SIGGRAPH Computer Graphics 39(1), 12 $16(2005)$

15. Thompson, D.J., Baranowski, T., Buday, R., Baranowski, J., Juliano, M., Frazior, M., Wilsdon, J., Jago, R.: In Pursuit of Change: Youth Response to Intensive Goal Setting Embedded in a Serious Video Game. Journal of Diabetes Science and Technology 1(6), 907-917 (2007)

16. Ye, Z.: Genres as a Tool for Understanding and Analyzing User Experience in Games. In: CHI 2004 Extended Abstracts on Human Factors in Computing Systems, Vienna, Austria, pp. 773-774 (2004)

17. Dyck, J., Pinelle, D., Brown, B., Gutwin, C.: Learning from Games: HCI Design Innovations in Entertainment Software. In: Graphic Interface, Halifax, pp. 159-169 (2003)

18. Aoki, N., Ohta, S., Okada, T., Oishi, M., Fukui, T.: INSULOT: A Cellular Phone-Based Edutainment Learning Tool for Children with Type 1 Diabetes. Diabetes Care 28(3), 760 (2005)

19. Cox, D.J., Gonder-Frederick, L.A., Kovatchev, B.P., Julian, D.M., Clarke, W.L.: Progressive Hypoglycemia's Impact on Driving Simulation Performance. Diabetes Care 23, 163-170 (2000)

20. Cox, D.J., Penberthy, J.K., Zrebiec, J., Weinger, K., Aikens, J.E., Frier, B.: Diabetes and Driving Mishaps: Frequency and Correlations from a Multinational Survey. Diabetes Care 26, 2329-2334 (2003)

21. Defazio, J.: An Innovative Approach for Developing Multimedia Learning Modules. In: ISECON 2001, Cincinnati, vol. 18, p. 34 (2001)

22. Faiola, A.: Designing Humane Technologies: A Potential Framework for HumanComputer Interaction Design. The International Journal of the Humanities 2(3), $1877-1886$ (2006) 